\title{
Situational Language Teaching in Ubiquitous Learning Environments
}

\author{
Angus F.M. Huang \\ Institute of Information Science, Academia Sinica, 128 Academia Road, \\ Section 2, Nankang, Taipei 115, Taiwan. \\ E-mail: angusfuminghuang@gmail.com

\section{Stephen J.H. Yang*} \\ Department of Computer Science and Information Engineering, \\ National Central University, No.300, Jhongda Rd., Jhongli City, \\ Taoyuan County 32001, Taiwan \\ E-mail: jhyang@csie.ncu.edu.tw
}

\section{Gwo-Jen Hwang}

Graduate Institute of Digital Learning and Education, National Taiwan University of Science and Technology, 43, Sec.4, Keelung Rd., Taipei, 106, Taiwan

E-mail: gjhwang.academic@gmail.com

*Corresponding author

\begin{abstract}
Situational language teaching (SLT) is an effective instruction paradigm for English teaching in terms of providing vocabularies and sentence patterns with their frequent situations through learning materials. With the growth of educational technology, we need powerful and suitable techniques to embody SLT's features in ubiquitous learning (u-learning) and thus to benefit teachers and learners. Although researchers have proposed several innovative types of u-learning scenarios, the improved SLT paradigm in u-learning environment has been rarely investigated. This study indicated a framework of ubiquitous learning school to promote the concept of u-learning and employ SLT pedagogy in u-learning environment; it is called U-SLT. In order to support its innovation and provide situational learning services on demand, situational mashups was suggested to identify learners' situation and learning requirement by means of integrating situation awareness with service mashups. The comparison between two u-learning modes, learning with situational mashups and learning without situation awareness support, were discussed. Experimental results showed that the students with the situational mashups support had a better learning performance and improved behaviours. Therefore, situational mashups was perceived to be a useful and desirable system for supporting U-SLT as well as the fundamental issue of a ubiquitous learning school.
\end{abstract}

Keywords: Ubiquitous Learning, Situational Language Teaching, Mashups, Situation Awareness, Ubiquitous Situational Language Teaching. 
Biographical notes: Dr. Angus F.M. Huang received the B.S. degree in the department of information management from the Yuan Ze University in 2003 , and the M.S. degree in network learning technology from the National Central University in 2005, both in Jungli, Taiwan. He received the Ph.D. degree in computer science and information engineering from the National Central University. He is a postdoctoral researcher in the Institute of Information Science, Academia Sinica, Taiwan. His current research interests concerns software engineering, knowledge engineering, instructional technology, information management, pervasive computing, and prediction market.

Dr. Stephen J.H. Yang is a Distinguished Professor of Computer Science \& Information Engineering, and the Associate Dean of Academic Affairs at the National Central University, Taiwan. Dr. Yang received his Ph.D. degree in Electrical Engineering \& Computer Science from the University of Illinois at Chicago in 1995. His research interests include knowledge management, Second Life, mobile learning, Web 2.0, semantic Web, social networks, context aware \& ubiquitous learning.

Gwo-Jen Hwang is currently a Chair Professor in the Graduate Institute of Digital Learning and Education at National Taiwan University of Science and Technology. In 1991, Dr. Hwang received his Ph.D. degree in Computer Science and Information Engineering from National Chiao Tung University in Taiwan. His research interests include mobile and ubiquitous learning, computer-assisted testing, expert systems and knowledge engineering. Dr. Hwang has published more than 330 academic papers, including 118 papers in such professional journals as Computers \& Education, Educational Technology \& Society, British Journal of Educational Technology, Innovations in Education and Teaching International, and The Electronic Libraries among others. Owing to the good reputation in academic research and innovative inventions of e-learning, in 2007, he received the annual Most Outstanding Researcher Award from the National Science Council in Taiwan.

\section{Introduction}

Because of the growth of educational technology, many studies were devoted to improving instruction paradigm with advanced devices or various learning processes. Situational language teaching (SLT) is a term not commonly used today, but it is an approach developed by British applied linguists in the 1930s to the 1960s (Richards \& Rogers, 1986). The instruction paradigm impacts language courses and teaching activities by its continuous use today. The structural view of a language is the basis for the oral approach and SLT. Speech as the basis of language and structure is regarded as the heart of the speaking ability (SIL, 1999). The instruction paradigm emphasizes that language structures must be presented in situations in which they could be used, implying its distinctiveness to SLT. Among the SLT activities, lecturers teach English vocabularies and sentence patterns in frequent situations through books, learning materials, photos, body language, fictitious scenarios. Teachers do not explain grammars and interpret sentences directly. Students must understand situations and apply the language and implied meaning to practice the related learning activities, e.g. repetition, written examination, dictation, oral conversation, and extended reading.

Beyond desktop learning, knowledge sources and learning experiences exist everywhere. Many effective e-learning materials not only appear on desktop but rise in 
ubiquitous environments, e.g. museum resources, nature observations, distance education, social contacts, medium transmission and personal experience. Many studies utilized context-aware techniques to identify learners' environments and requirements or to assist learners in acquiring information from the physical world (Silius, Miilumäki, Huhtamäki, Tebest, Meriläinen \& Pohjolainen, 2010; Yang 2006; Hwang, Tsai \& Yang, 2008). This paradigm of getting learning services anywhere anytime is called ubiquitous learning (ulearning). These contributions promote various human-environment interactions and enhance learners' knowledge cognition from the real world (Graf, Yang, Liu \& Kinshuk, 2009).

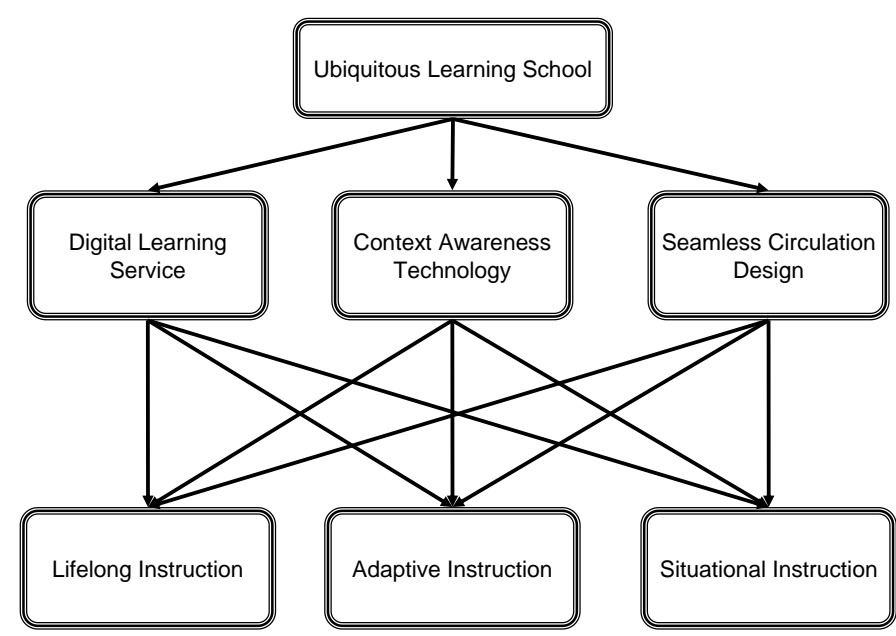

Figure 1. Framework of a Ubiquitous Learning School

The needs of $\mathrm{u}$-learning must take the functional and cognitive requirements into account from learners and teachers' perspectives. Therefore, context awareness or situation awareness is one kernel concept to achieve the u-learning process, such as environment information, learner capability, learning goals and so on (Vreman \& Jong 2006; Teo \& Chai, 2009). To clearly popularize u-learning in school even in daily life, the authors suggested a framework of a ubiquitous learning school as shown in Figure 1. Digital learning service, context awareness technology, and seamless circulation design will construct the foundation of a ubiquitous learning school. Meanwhile, the u-learning school supports the lifelong instruction, adaptive instruction, and situational instruction. From the SLT viewpoint, programs such as news, soap operas and documentaries have the potential to enhance the learners' language experience by showing the target language, culture and context of use (Fallahkhair, Pemberton, \& Griffiths, 2007). However, SLT does not smoothly achieve relevant learning activities in the ubiquitous learning school for the digital learning services and human-environment computing paradigm. Accordingly, this paper proposes the Ubiquitous SLT and achieves it by situational mashups.

Based on the vision of a u-learning school and the concept of situational language teaching, the authors define a new SLT paradigm in the u-learning environment. Ubiquitous SLT (U-SLT) aims at authoring teachers' SLT-based learning activities in a digital learning form and to publish them on the Internet or deploy them on the 
ubiquitous computing environment. Along with the teaching support, students can obtain situational learning according to teachers' SLT-based pedagogy. Based on the scenario, students can understand the meaning and knowledge of the learning goal from multiple learning resources. U-SLT learners are people who use ubiquitous learning services on portable computing devices to proceed with SLT learning activities. They need a uniform and convenient platform to receive situational learning services, e.g. course slides, video conference, on-line dictionary, instant messaging, game-based learning guidance, and so on. The goal of U-SLT is to achieve SLT pedagogy in a u-learning environment that accounts for student individuality and learning situations.

With the evolvement of software engineering, ubiquitous computing and Web 2.0, the support of end user programming becomes more desirable. In addition to the silver bullet of heterogeneous systems integration solution, Web services, Web 2.0 initiated the civilian programming environment (Yang, Okamoto \& Tseng, 2008). The rising popularity of user-driven web applications, including Blogs, Wikis, Mashups, RSS, Podcast, Social Networks, P2P networking and Communities, has reflected the aspired initiative of users. Enterprises utilize relevant technologies in Web 2.0 not only for sharing daily thought, but for also for communicating with business partners, improving customer service, integrating suppliers, and managing knowledge internally. Most importantly, mashups provide a universal solution for users with a non-IT background to program specific applications. The Mashups component is a widget. Widget can be operated with a pure program or invoke other Web services. Some popular Mashups platforms work on the Internet, such as iGoogle (http://www.google.com/ig), MyYahoo (http://my.yahoo.com), Hinet Xuite (http://www.xuite.net), Yahoo! Pipes (http://pipes.yahoo.com), Microsoft Popfly (http://www.popfly.ms), and Windows Live (http://www.live.com) as shown in Figure 2. Although all of these tools provide some kind of support for end user programming, none of them focuses on context awareness and their design does not support situational mashups. Automatically composing necessary situational mashups is important, when the end user roams in a ubiquitous computing environment and executes different tasks, such as ubiquitous learning and real-time business. The authors believe that the mobility and flexibility of the mashups technique can assist in executing situational instruction.

Situational mashups has been addressed in other studies. For example, QEDWiki is a wiki-based mashups software published by IBM (QEDWiki, 2007). However, to the best of our knowledge there are currently no environments that are designed specifically to support context awareness and automatic composition in situational mashups. In order to better achieve context awareness and facilitate situational mashups the authors firstly developed a context-aware SOA-based mashups system based on researching into mashups in Web 2.0, context awareness, end user programming by applying their vision on situational mashups activities.

The general purpose of this study was to develop a situational mashups system and achieve the pedagogy of situational language teaching in a u-learning environment. In this study the authors' main concern was how context-aware mechanism supports automatic situational mashups and how this mechanism can best be improved. To formularize this study, the design problems are defined as follows:

1. How should a context awareness mechanism be developed to facilitate situational mashups?

2. How to describe students' situation and the related context information?

3. How to realize the students' situation and learning requirements? 
4. How to offer the required situational learning services?

5. How to choose the optimal services composition based on the multiple context constraints?

6. How does such a situational mashups support situational language teaching in a u-learning environment?

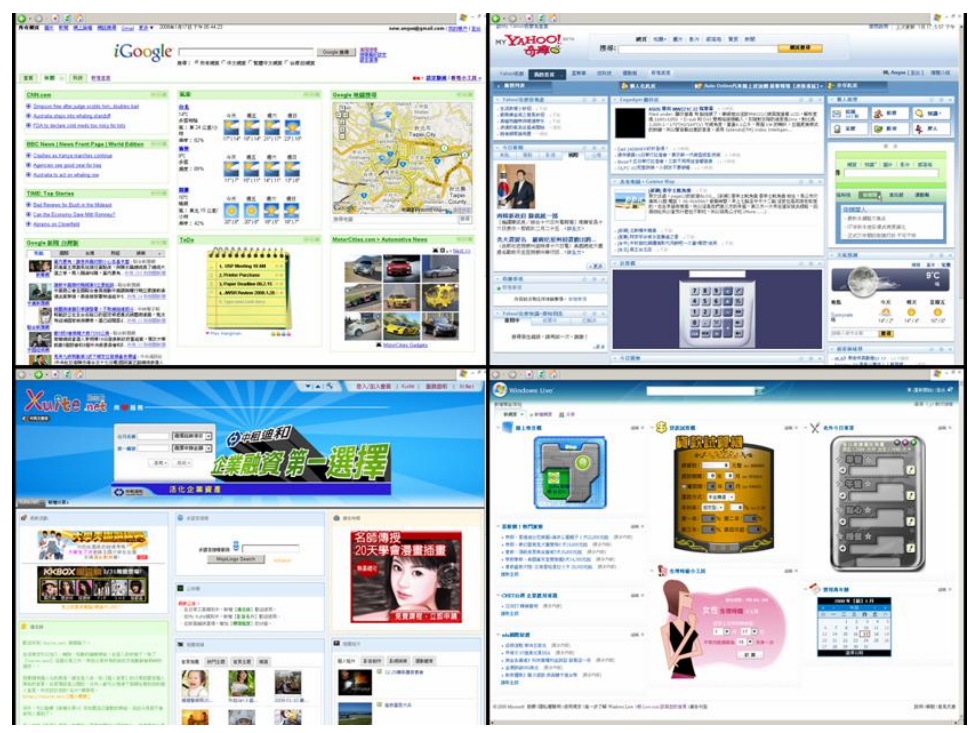

Figure 2. Four popular mashups platforms in Internet, they are iGoogel, MyYahoo, Hinet Xuite, and Windows Live.

The authors categorize the primary characteristics of SituMash as follows: (1) Situation awareness- it can interpret student's context factors and reason students' specific situation. (2) Automatic composition- it can compose student's necessary mashups with its planning. (3) Service orientation- it uses service as the software basis that students are need not to build the kernel components. (4) End-user autonomy-it allows students to set the configuration of mashups with their favorites or habits. (5) Single platform- it proposes a universal platform to avoid conflicts between heterogeneous systems and to provide an integrated using experience. Finally, the implementation of the SituMash system is discussed in detail, followed by results derived from applications and evaluation, which the situational mashups has performed successfully.

In this paper the authors concentrate on the design and development of a situational mashups system for ubiquitous situational language teaching in a ubiquitous learning school. The system aims to employ the strengths of situation awareness and service mashups not only to identify the learners' situation and learning requirements but also to provide situational and recommended learning services. Ubiquitous SLT that takes advantage of situation awareness and service mashups is the primary focus of the Situational Mashups. The experiments showed that the situational mashups was perceived to be a useful and desirable system to support ubiquitous situational language teaching and also the fundamental issue of a Ubiquitous Learning School.

This paper is organized as follows. Firstly, the authors propose the research questions and observe related literature. Then, the system design and research 
methodology is introduced. After the implementation, the system efficiency is evaluated. Finally, the authors conclude the work and suggest further steps.

\section{Literature Review}

The concepts of situational computing, ubiquitous learning, and Web 2.0 and mashups trigger the improvement of ubiquitous situational language teaching that this paper proposes. This section discusses the related developments and the literature review.

\subsection{Situational Computing}

Situational Computing is an advanced concept in assisting people better proceed with daily work and problem solving. Situations use a series of participant, activity, time, location, equipment to express user's specific state. The situational computing evaluates one user's state and provides him or her with the necessary services. There are several researches devoted to the study of situation detection so far. The core technology is Context Awareness (Dey, Salber \& Abowd, 2001). Context awareness is used to detect, represent, and inference the state and behavior of users. Up to now, it has focused on various studies pointing out the academic and industrial applications for a humancomputer interaction environment. Context awareness supports several applied sciences, such as health-care system, recommendation system, sensor network, smart environment, and life long learning. Building situational system is a complex, dynamic and iterative process, especially for a busy businessman or a mobile user facing various activities in a running trigger of events. Manifold context awareness mechanisms are achieved for users. A complete system development tool kit for building context-aware applications (Loke, 2006) and the mechanism of analyzing the uncertainty of context information (Ranganathan, Al-Muhtadi, \& Campbell, 2004) were proposed. However, these studies do not provide support for end user programming towards the opportunity for an autonomous service selection.

\subsection{Ubiquitous Learning}

Microsoft Research proposed a new class of applications that relies on real-time sensor data and its mash-up with the geocentric Web to provide instantaneous environmental visibility and timely decision supports. They challenged the data publishing, scalable data management, data visualization and sensor discovery issues to make users can take advantage of the portal and tools to make queries over live data sources. In order to extend the potential of SensorMap, they also provide the mashup APIs. Users can mash up the online live data sources with other applications. It is powerful. Nevertheless, it is not easy for end-users to compose these applications. A context-interactive application (Tan, Liu, \& Chang, 2007), based on radio frequency identification (RFID), Internet, ubiquitous computing, and embedded system, has been developed. This system provides a mobile-based interactive learning environment (MOBILE) server for teachers and mobile-tools (m-Tools) for students. Teachers can achieve their outdoor teaching pedagogy effectively by providing the context-aware expert guidance and outdoor learning tools. Students can observe outdoor conditions and materials attentively; further, get the useful knowledge with these technologies.

Microsoft Research proposed a new class of applications that relies on real-time sensor data and its mash-up with the geocentric Web to provide instantaneous environmental visibility and timely decision supports. They challenged data publishing, scalable data management, data visualization and sensor discovery issues to make users take advantage of the portal and tools to query over live data sources. In order to extend 
the potential of SensorMap, they also provide the mashup Application Programming Interface. Users can powerfully mash up the online live data sources with other applications. Nevertheless, it is not easy for end-users to compose these applications. A context-interactive application (Tan, Liu, \& Chang, 2007), based on radio frequency identification (RFID), Internet, ubiquitous computing, and embedded system, has been developed. This system provides a mobile-based interactive learning environment (MOBILE), server for teachers and mobile-tools (m-Tools) for students. Teachers can achieve their outdoor teaching pedagogy effectively by providing the context-aware expert guidance and outdoor learning tools. Students can observe outdoor conditions and materials attentively; further, students have access to useful knowledge by the use of these technologies.

\subsection{Web 2.0 and Mashups}

A comprehensive survey of Web 1.0 applications results in the fact that they are mainly database driven. Developers build an application logic to manipulate the databases. From the original database schema, web surfers can obtain rich information. However, these applications are centralized and rely on their own relational database, limiting the possibilities for data integration. Mashups is a rapid growing Web development paradigm and provides rich user experience. The mechanism can assist in combining different Web applications through supported technologies, such as Ajax, SOAP, REST, screen scraping, Semantic Web, RDF in a succinct program development way. Duane Merrill offers a brief survey of the prominent mashup genres (Merrill, 2006). These are mapping mashups, video and photo mashups, searching and shopping mashups and news mashups at present. For example, one of the big catalysts for the appearance of mashups was the Google's project, called Google Maps API. Another popular application is the news mashups. Syndication feed mashups (Lindahl \& Blount, 2003) can aggregate user's feeds and present them over the Web, creating a personalized newspaper that plays according to user's interests or domain. MashMaker assists non-expert users in creating their own mashups easily based on data and queries proposed by other users and by remote sites (Ennals \& Garofalakis, 2007). They encourage users to find information by exploring, rather than by writing queries. Users can share data, widgets, and widget recommendation collaboratively especially. Although they apply the social network to improve the mashups selection, users have to explore those candidates by themselves eventually.

\section{The Situational Mashups System}

The SituMash system supports the situational mashups composition according to the students' context and teacher's teaching goal. The situational mashups environment is illustrated in Figure 3. There are five parts in such environment, student, computing device, SOA, mash hub and physical environment. Students will handle some computing devices and roam at different deployed by service providers. These learning services also can be invoked by the widgets; therefore, a more convenient software paradigm is achieved. Students also can arrange the mashups according to their requirements. In order to achieve more powerful applications and reduce the interruption to students, the authors propose the SituMash system plugging in the mash hub. The SituMash system can compose widgets according to student's situation automatically. Students can move to different locations and thereby get the specific learning service for U-SLT activities (as shown in Figure 4). The specific context constraints can lead to students' specific situation and the situation will then trigger the system to recompose the necessary widgets for the students. 


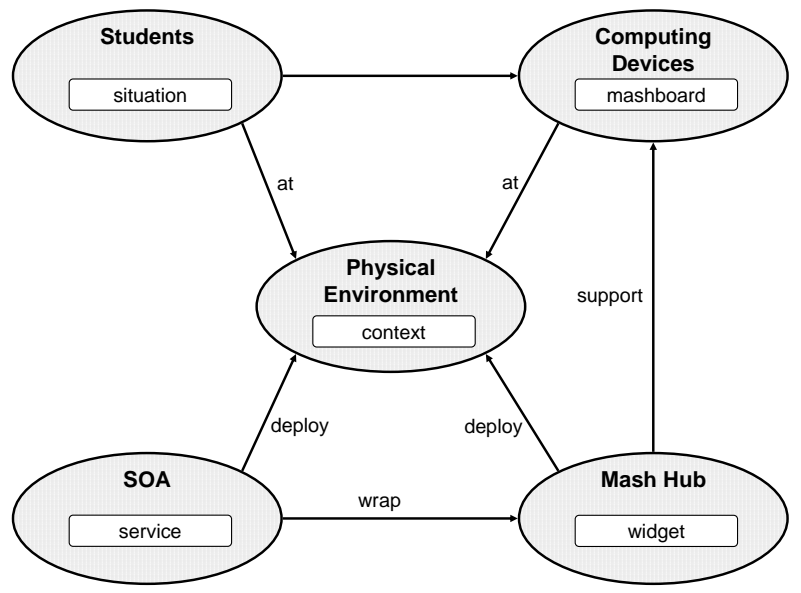

Figure 3. Situational mashups environment.
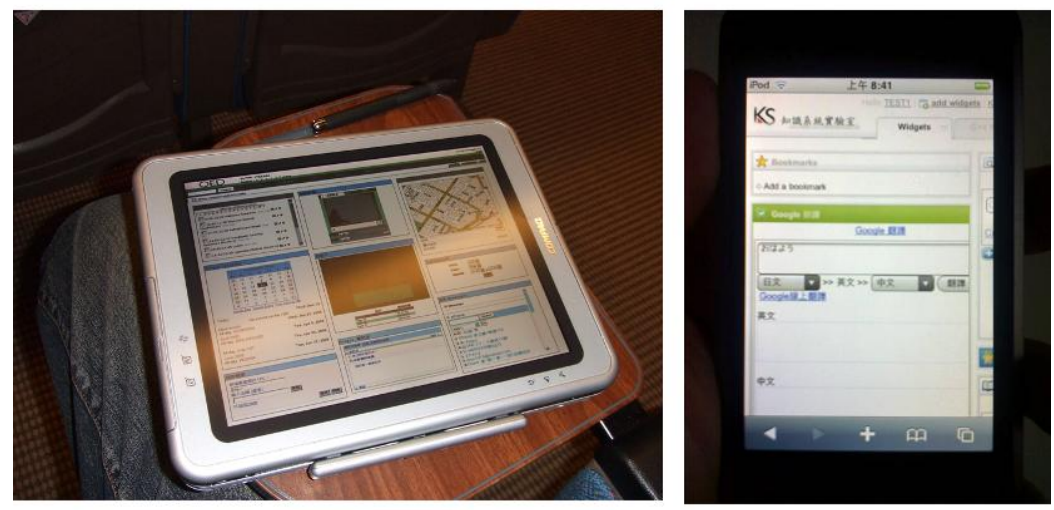

Figure 4. An example of Situational mashups that composes different learning services in different contexts such as device, location, and task)

\subsection{Identifying Requirements}

In order to provide students with the situational services when they roam in different situations, the authors designated the following basic concerns that should be provided by the SituMash system: (1) When to detect the student's context? (2) How to reason the student's situation? (3) How to determine the necessary widgets for the specific situation? (4) How to select the optimal widgets from numerous candidate widgets? (5) How to compose the selected widgets dynamically?

The situation reasoning includes many inference rules. Considering the complexity of these rules, the students may not be aware of them when the system reasons the students' situations. The SituMash system should infer the specific situational widgets by the students' context model quietly. One approach could be to design the system to determine the necessary widgets autonomously and compose them automatically for students' uses. The disadvantage of this approach is that students might have their own opinions and disagree with the recommendation. Another approach could be to allow the students to modify the widgets. The authors anticipate that the situation 
reasoning mechanism will reason the students' situation appropriately and the service inference mechanism will infer the necessary widgets suitable for the situation.

The SituMash consists of the following components: Context Modeling to build a knowledge model to represent the student's context factors. Situation Reasoning to reason the student's situation through the context model with the situation inference rules. Service Inference to infer the situational widgets through the student's situation with the service inference rules. Mashups Optimization to compose the final widgets in an optimal way towards the minimal costs and the maximal benefit.

\subsection{Context Representation}

In order to provide situational mashups for designing support in a ubiquitous computing environment the authors go deep into the study of context representation. A common definition of context points towards a five tuple \{people, activity, time, location, object\}. However the authors argued that the definition is too vague and widespread. The characteristics of such model may let it be driven to futility. Therefore, the refined context definition addresses a Context Factor \{Social, Event, Schedule, Location, Equipment $\}$ to reflect the usage of a mobile and ubiquitous computing environment. This model appears clearer and without a hitch to represent the students' context. And it will make the situation reasoning mechanism go smoothly in reasoning the students' situation from a context factor. The social factor addresses the people interacting with students; it will assist in reasoning the students' situation through the interactive people's professional title. The event factor refers to the activity executed by the student. The schedule means the students' personal schedule; it will assist in understanding the students' assignments in a specific duration. The location factor is a popular context factor in this research area; it can indicate the students' working state through a corresponding location information. The equipment factor addresses the devices surrounding the students; it may limit and shape the usage of information acquisition. This context model can be represented in a formal description (Yang \& Chen, 2008). Subsequently, the formal context information can be used to reason the situation in the following step.

\subsection{Situation Reasoning}

Advanced research about service provision can obtain student profiles to promote the satisfaction of the users experience. Nevertheless, changing and dynamic student situation were not considered by now. They can not infer and predict the student's specific situation, therefore they cannot propose applicable services to students. When using context awareness, the goal of the SituMash system is to create a seamless and intelligent Web experience for the students by composing necessary widgets with a workflow planning rather than selecting the widgets manually. Based on previous research result (Yang, Zhang \& Chen, 2008), this study used the rule-based system called JESS to construct a situation reasoning mechanism. A context interpretation rule base has been proposed. According to the knowledge of context interpretation, the system can predict students' situations such as to attend a meeting, to attend a class, to operate a vehicle, to join outdoor learning, to visit a museum, and so on. Therefore, the SituMash system can use this situation information to plan the suitable and necessary tasks towards the students' goals. The automatic mechanism also can avoid too many manual students' inputs by using extra situation information. It is important to rely on the users using experience in a ubiquitous environment. 


\subsection{Workflow Planning}

Situational-mashups systems for ubiquitous-computing environments are an emerging trend in service science. But there is a problem due to the changeable states of the situations in ubiquitous computing environments. From the characteristics user mobility, device variety, time validity, location dependence, students require special solutions that address different mashups guidelines. The set of potentially available mashups widgets varies because of the above-mentioned characteristics, so it cannot be forecasted and preplaned. Situational Mashups should be designed to provide students with the opportunity of minimum manual inputs of context to achieve the situational tasks in an automatic widgets composition. The authors consider the widgets configuration as a workflow planning (Thompson, Li, and Xiao, 2007). The user request is a set of inputs, the user context is a constraint, and the user's goal is a set of desired outputs. The widgets are similarly treated as operators. Thus, planning skills can be used, and a special search that uses local knowledge of action operators and constraints to find a set of widgets that will satisfy the inputs (user request) and outputs (desired goal). In the SituMash system, the Partial Order Planning (POP) algorithm contributes to automatically finding the necessary widgets.

\subsection{Mashups Optimization}

It is possible to receive many functional-matching widgets as a result of the workflow planning mechanism in the widgets repository. Selecting one optimum combination from the tasks set is an important and difficult issue. From the software point of view, each widget has its own characteristics, such as cost, response time, and reliability. From the hardware point of view, each each widget has its own point of view, such as device computing capability, memory, operating style, and mobility. Finally, from the learning methodology point of view, authorization, learning age, and course dependency are important characteristics. In addition, software engineering and ubiquitous computing concerns play also a role. To optimize the widgets composition according to the limitation of software and hardware capabilities and students' requirements is the goal of the mashups optimization mechanism. This classical software requirement optimization also has been investigated for a long time. There have been various optimization solutions proposed, such as mathematical programming, genetic algorithm, ant colony optimization, cultural algorithm, and particle swarm optimization. In search-based software engineering (SBSE) research area (Harman \& Jones, 2001), there are many contributions to combinational optimization based on the meta-heuristic mechanisms. The PSO algorithm is a meta-heuristic methodology, which was designed from the natural observation of birds seeking for food. This algorithm requires only original mathematical operators and profits from the need for less computation memory and quick computing speed (Kennedy \& Eberhart, 1995). The sharing of information among participants offers an evolutionary advantage. This insight was essential for the particle swarm optimization. This study is based on the PSO algorithm to compute and customize the optimal mashups composition through the parameters and fitness function designated by students. Finally, students will obtain the situational and optimal mashups for their goals in the mashboard starting from the situation reasoning to Efficiency is workflow planning to mashups optimization. 


\section{Evaluation and Discussion}

To evaluate the effectiveness of this study, the students' perceived satisfaction, the teachers' perceived satisfaction, and the students' learning outcome are discussed in this section.

\subsection{Evaluation of the Students' Perceived Satisfaction}

The authors made a series of experiments with the SituMash system to demonstrate the satisfaction with this system and to explore some phenomena of situational computing. Students in both groups with the SituMash system (with situational computing) and with the QEDWiki system (without situational computing) were asked about their perceptions of the situational language learning activities. Students were asked to fill in a questionnaire (Table 1) by checking the answers on a Likert-type 5-point scale. 320 students completed the questionnaire, among which, 160 students belonged to the SituMash group and 160 students belonged to the QEDWiki group. This study compares the students' attitudes between the two groups with t-test statistics. The results are shown in Table 2.

In this experiment, the SituMash group students showed more positive perceptions about the situational language learning activities than the QEDWiki group students $(42.50 \%$ SA vs. $26.88 \%$ SA, $28.13 \%$ A vs. $26.25 \%$ A, Table 2). Also more QEDWiki group students disliked the learning activities $(11.25 \%$ D vs. $21.88 \%$ D, $4.38 \%$ $\mathrm{SD}$ vs. $7.50 \% \mathrm{SD}$, Table 2). The t-test results revealed that there were significant differences in the students' attitudes between the two groups. The SituMash group liked situational language learning better (Question 1, $\mathrm{t}=13.22, \mathrm{p}<0.05$ ). Positively, more SituMash students felt the SLT learning activities are helpful for language learning (Question 2, $\mathrm{t}=14.76, \mathrm{p}<0.05$ ). The situational learning services composition assists students in understanding the learning objectives constructively (Question 3, $\mathrm{t}=10.42$, $\mathrm{p}<0.05)$. In addition, the interaction with learning services in SituMash group is better than QEDWiki (Question 4, $\mathrm{t}=17.04, \mathrm{p}<0.05$ ). However, no significant differences were found between the two groups regarding the interactions with classmates and teacher, and the interaction efficiency are bad both (Question 5, $\mathrm{t}=6.67, \mathrm{p}=0.1545$, Mean $=2.9625$ and 2.6875). The authors conjectured that the social interaction is influenced by the design of the learning services and the personality of the students. Furthermore, it was observed that the situation reasoning, workflow planning, and mashups optimization can reduce the loading of the students' manual operation. Therefore, the results also showed that the SituMash group indicated that they had better interactions with the platform (Question 6, $\mathrm{t}=16.81$, $\mathrm{p}<0.05$, Mean=3.9 vs. 3.5).

The authors found an interesting phenomenon thanks to the evaluation of the interviews. Students answers convinced that the SituMash system can effectively economize the configuration time, because they do not need to select the widgets manually. The system can automatically arrange the suitable composition of mashups for the specific situation that allows them to proceed with the tasks seamlessly. Even the students felt that the tasks planning mechanism can redeem the ignorance that what tools they should work on classes. Although the supply of software is rich, students informed that if the test can cope with other devices they would have felt more convenient, such as PDA, smart phone, widget player, or thinner Tablet PC. Moreover, students indicated that the user experience of the pure QEDWiki system refers to inconvenience and troubles. Summarizing the results, the situation reasoning and the tasks planning mechanisms can advance the situational and pervasive computing domain. 
Table 1. Questions about the students' perceived satisfaction

\begin{tabular}{cl}
\hline Questions & Question Sentences \\
\hline 1 & I like U-SLT learning activities. \\
\hline 2 & The U-SLT learning activities are helpful. \\
\hline 3 & I feel easy to understand each learning objective. \\
\hline 4 & Interact with learning services is very easy. \\
\hline 5 & I had good interactions with classmates and teacher. \\
\hline 6 & I had good interactions with the platform.
\end{tabular}

Table 2. The students' perceived satisfaction of U-SLT

\begin{tabular}{|c|c|c|c|c|c|c|c|c|c|}
\hline $\bar{Q}$ & Group & $\mathrm{SA}(\%)$ & $\mathrm{A}(\%)$ & $\mathrm{N}(\%)$ & $\mathrm{D}(\%)$ & $\mathrm{SD}(\%)$ & Mean & $t$ & $p$ \\
\hline \multirow{2}{*}{1} & SituMash & 42.50 & 28.13 & 13.75 & 11.25 & 4.38 & 3.9313 & \multirow{2}{*}{13.22} & \multirow{2}{*}{0.0102} \\
\hline & QEDWiki & 26.88 & 26.25 & 17.50 & 21.88 & 7.50 & 3.4313 & & \\
\hline \multirow{2}{*}{2} & SituMash & 51.88 & 28.13 & 15.00 & 3.75 & 1.25 & 4.2563 & \multirow{2}{*}{14.76} & \multirow{2}{*}{0,0052} \\
\hline & QEDWiki & 36.25 & 26.25 & 23.13 & 9.38 & 5.00 & 3.7938 & & \\
\hline \multirow{2}{*}{3} & SituMash & 26.88 & 47.50 & 15.00 & 7.50 & 3.13 & 3.8750 & \multirow{2}{*}{10.42} & \multirow{2}{*}{0.0340} \\
\hline & QEDWiki & 20.00 & 37.50 & 23.13 & 14.38 & 5.00 & 3.5313 & & \\
\hline \multirow{2}{*}{4} & SituMash & 33.13 & 45.00 & 15.00 & 4.38 & 2.50 & 4.0188 & \multirow{2}{*}{17.04} & \multirow{2}{*}{0.0019} \\
\hline & QEDWiki & 20.00 & 37.50 & 30.63 & 8.13 & 3.75 & 3.6188 & & \\
\hline \multirow{2}{*}{5} & SituMash & 15.00 & 21.25 & 23.13 & 26.25 & 14.38 & 2.9625 & \multirow{2}{*}{6.67} & \multirow{2}{*}{0.1545} \\
\hline & QEDWiki & 8.13 & 15.63 & 30.00 & 29.38 & 16.88 & 2.6875 & & \\
\hline \multirow{2}{*}{6} & SituMash & 26.25 & 50.63 & 15.00 & 3.13 & 5.00 & 3.9000 & \multirow{2}{*}{16.81} & \multirow{2}{*}{0.0021} \\
\hline & QEDWiki & 15.63 & 48.13 & 14.38 & 14.38 & 7.50 & 3.5000 & & \\
\hline
\end{tabular}

Note. SA: strongly agree; A: agree; N: neutral; D: disagree; SD: strongly disagree.

$* p<0.05$

\subsection{Evaluation of the Teachers' Perceived Satisfaction}

To demonstrate whether the SituMash system can satisfy the original intention of situational language learning, this study also asked six teachers to give their perceptions of using the system and its functions in the seven questions shown in Table $3.83 .33 \%$ of the teachers agreed that the user interface of the SituMash system was clear and straightforward (Question 1). 83.33\% reflected that the learning services appearance in U-SLT can keep the original intention of traditional SLT (Question 2). Positively, a majority of teachers agreed that the context representation, the situation reasoning, and the workflow planning are important, and precise and helpful for SLT learning activities (Question 3, 50\%A; Question 4, 33.33\% SA; Question 5, 50\% SA). However, teachers doubted that mashups optimization can affect learning services composition effectively. $66.67 \%$ teachers disagreed its effectiveness. Overall, teachers are not confused with the use of the SituMash system (Question 6, 33.33\% SA, 50\% A, 16.67\% N). 
This result showed a positive evaluation of the SituMash system for assisting teachers in executing situational language teaching. According to the teachers' profession in the SLT pedagogy, the survey revealed that the ubiquitous situational language teaching can be achieved successfully in a u-learning environment by the SituMash system. Teachers expressed that improving the SLT in such a u-learning environment is interesting and can enrich the learning activities through their empirical observation. In addition, the U-SLT assured teachers of using an effective teaching/learning paradigm. They believe that the physical interaction and the real experience can deepen the students' understanding of knowledge cognitively.

Table 3. The Teachers' perceived satisfaction of U-SLT

\begin{tabular}{|c|c|c|c|c|c|c|c|}
\hline \# & Question & $\mathrm{SA}(\%)$ & $\mathrm{A}(\%)$ & $\mathrm{N}(\%)$ & $\mathrm{D}(\%)$ & $\mathrm{SD}(\%)$ & Mean \\
\hline 1 & $\begin{array}{l}\text { The user interface of the SituMash } \\
\text { system is clear and straightforward. }\end{array}$ & 50.00 & 33.33 & 16.67 & 0.00 & 0.00 & 4.33 \\
\hline 2 & $\begin{array}{l}\text { Are the learning services invoked } \\
\text { by the SituMash system in a U-SLT } \\
\text { conform to the original intention of } \\
\text { a traditional SLT? }\end{array}$ & 33.33 & 50.00 & 16.67 & 0.00 & 0.00 & 4.17 \\
\hline 3 & $\begin{array}{l}\text { Is the context representation } \\
\text { mechanism of the SituMash system } \\
\text { suitable to describe the learning } \\
\text { context in a SLT? }\end{array}$ & 0.00 & 50.00 & 50.00 & 0.00 & 0.00 & 3.50 \\
\hline 4 & $\begin{array}{l}\text { Is the situation reasoning } \\
\text { mechanism of the SituMash system } \\
\text { suitable to infer the students' } \\
\text { learning situation for a learning } \\
\text { objective? }\end{array}$ & 33.33 & 50.00 & 16.67 & 0.00 & 0.00 & 4.17 \\
\hline 5 & $\begin{array}{l}\text { Is the workflow planning } \\
\text { mechanism of the SituMash system } \\
\text { suitable to effectively plan the SLT- } \\
\text { based learning services? }\end{array}$ & 50.00 & 50.00 & 0.00 & 0.00 & 0.00 & 4.50 \\
\hline 6 & $\begin{array}{l}\text { Is the mashups optimization } \\
\text { mechanism of the SituMash system } \\
\text { suitable to effectively compose the } \\
\text { required learning services by the } \\
\text { lowest learning costs? }\end{array}$ & 0.00 & 33.33 & 0.00 & 66.67 & 0.00 & 2.67 \\
\hline 7 & $\begin{array}{l}\text { I encountered technical problems } \\
\text { while using the SituMash system. }\end{array}$ & 33.33 & 50.00 & 16.67 & 0.00 & 0.00 & 4.17 \\
\hline
\end{tabular}

Note. SA: strongly agree; A: agree; N: neutral; D: disagree; SD: strongly disagree.

\subsection{Evaluation of the Learning Outcome}

One of the important research questions in this study is that: Does the U-SLT improve the learning outcomes? The authors designed this experimental research to demonstrate the learning effectiveness of this study. In this experiment, 30 students were randomly chosen from the high-level English communication junior course for the Experimental Group, and 30 students for the Control Group. The Experimental Group proceeded with the U-SLT-based learning and the Control Group proceeded with the SLT-based learning. Before the experiment, a pre-test was conducted to compare the prerequisite knowledge of the students in both groups; after the experiment activities, a post-test was performed to evaluate their subsequent learning outcomes. 


\section{Pre-test}

The pre-test aimed to ensure that both groups of students had the equivalent prerequisite knowledge required for taking the course. The examination questions of the pre-test included 30 multiple-choice test items and ten open-ended test items. Table 4 presents the t-test results of the pre-test. Notably, the mean and standard deviation of the pre-test was 82.4 and 5.87 for the Experimental Group, and 83.233 and 5.864 for the Control Group. The p-value indicates that the two groups do not significantly differ at the 0.05 level. It is evident that the two groups of students had statistically equivalent abilities in completing the programming course.

Table 4. Pre-test $t$-value and descriptive statistics for the two groups

\begin{tabular}{lccccc}
\hline & $\mathrm{N}$ & Mean & S.D. & $\mathrm{df}$ & $T$ \\
\hline Experimental Group & 30 & 82.400 & 5.870 & 58 & -.550 \\
Control Group & 30 & 83.233 & 5.864 & & \\
\hline
\end{tabular}

\section{Post-test}

The post-test was intended to compare the learning achievements of the two groups of students after taking the programming course. Table 5 lists the t-test values for the post-test results. Notably, the mean and standard deviation of the post-test were 92.5 and 5.283 for the Experimental Group, and 88.033 and 5.417 for the Control Group. Following the t-test results it can be concluded that the Experimental Group achieved significantly better performance than the Control Group after implementing the subject approach $(t=3.233, p<.001)$, implying that the innovative approach is effective.

Table 5. Post-test t-value, effect size and descriptive statistics for the two classes.

\begin{tabular}{lcccccc}
\hline & $\mathrm{N}$ & Mean & S.D. df & $t$ & $p$ & $d$ \\
\hline Experimental Group & 30 & 92.500 & 5.283 & 58 & $3.233^{* * *}$ & .002 .83 \\
Control Group & 30 & 88.033 & 5.417 & & & \\
\hline
\end{tabular}

$p<0.01$

\section{Conclusion and Further Work}

Human-computer interaction is changing gradually. Students do not need to be restrained by sitting in front of desktops and using the techniques of mobile computing and ubiquitous computing. The interactions between humans and computers can be more humanized because the computing units have fused into our physical environment. It means that everyone can benefit from the convenience brought by computers anytime and anywhere in the daily life. Based on the empirical study, the authors argue that a ulearning system should be easy to use and intuitive to get information in order to support learning and teaching in the platform, not just laborious in learning the platform itself. Furthermore, it should be optimal in learning services provision with learning factors and context that will get benefits from the perspective of cognitive learning theories. 
Situational language teaching is an effective instruction paradigm. However, in the generation of u-learning, we need powerful and suitable techniques to achieve its concepts and provide benefits to teachers and learners. Context constraints, such as partner, event, time, location, and equipment, situate people in a special learning situation. Being in different situations will confront different learning activities, and thereupon students will need some hybrid application system to support them. The application of situational computing is regarded as a situational mashups. With the contribution of this study, the student's situation can be detected by situational mashups. Thereby, situational mashups can accommodate to the students' activities in a ubiquitous environment, as well as augment the capability of Web 2.0 for ubiquitous computing.

The authors integrated the related techniques to achieve the U-SLT paradigm and promoted the concept of a ubiquitous learning school by finding that the colleagues or participants around one student using the situational mashups will influence the learning services configuration. The authors conjecture that the social networks of students will influence their social-based learning activities, regardless of positively or negatively. Therefore, more social network factors will be considered in situation reasoning. Following the growth of mashups, more convenient composition mechanisms will appear and more powerful platforms will be proposed. The usability and validity of the mashups configuration will become an important issue. The authors will also devote to the verification of mashups to make sure that the learners' artifacts are safe and usable, and extend the situational mashups to various pedagogies.

\section{Acknowledgements}

The authors would like to express their deepest thankfulness to Prof. Chin-Chung Tsai for his valuable and constructive comments to the early version of this paper. This work is supported by the National Science Council, Taiwan under grants NSC 98-2511-S-008006-MY3 and NSC 98-2511-S-008-007-MY3.

\section{References}

1 Dey, A.K., Salber, D. \& Abowd, G.D. (2001). A conceptual framework and a toolkit for supporting the rapid prototyping of context-aware applications. HumanComputer Interaction, 16(2), 97 - 166.

2 Ennals, R.J. \& Garofalakis, M.N. (2007) MashMaker: mashups for the masses. Proceedings of the 2007 ACM SIGMOD international conference on Management of data. 1116 - 1118.

3 Fallahkhair S., Pemberton L. \& Griffiths R. (2007). Development of a cross-platform ubiquitous language learning service via mobile phone and interactive television. Journal of Computer Assisted Learning, 23(4), 312 - 325.

4 Graf S., Yang G., Liu T.C. \& Kinshuk (2009). Automatic, Global and Dynamic Student Modeling in a Ubiquitous Learning Environment. Knowledge Management \& E-Learning: An International Journal (KM\&EL), 1(1), $18-35$.

5 Harman, M. \& Jones, B.F. (2001). Search-based software engineering. Information and Software Technology, 43(14), 833 - 839.

6 Huang, R.H.Y., Yang, S.J.H. \& Tsai, C.C. (2009). Web 2.0 for Interactive e-learning. Journal of Interactive Learning Environments, 17(4), 257-259. 
7 Hwang, G.J., Tsai, C.C. \& Yang, S.J.H. (2008). Criteria, Strategies and Research Issues of Context-Aware Ubiquitous Learning. Educational Technology and Society, 11(2). $16-26$.

8 Kennedy, J. \& Eberhart, R. (1995). Particle swarm optimization. IEEE International Conference on Neural Networks, 4:27, 1942 - 1948.

9 Lindahl, C. \& Blount, E. (2003). Weblogs: simplifying web publishing. IEEE Computer, 36(11), $114-116$.

10 Loke, S.W. (2006). Context-aware artifacts: two development approaches. IEEE Pervasive Computing, 5(2), 48 - 53.

11 Merrill, D. (2006). Mashups: The New Breed of Web App. http://www.ibm.com/developerworks/library/x-mashups.html

12 QEDWiki. (2007). http://services.alphaworks.ibm.com/qedwiki/

13 Ranganathan, A., Al-Muhtadi, J. \& Campbell, R.H. (2004). Reasoning about uncertain contexts in pervasive computing environments. IEEE Pervasive Computing, 3(2), $62-70$.

14 Richards, J. C. \& Rogers, T. S. (1986). Approaches and methods in language teaching: A description and analysis. Cambridge, UK: Cambridge University Press.

15 SIL International (1999). Situational Language Teaching. http://www.sil.org/lingualinks/languagelearning/waystoapproachlanguagelearning/S ituationalLanguageTeaching.htm

16 Silius K., Miilumäki T., Huhtamäki J., Tebest T., Meriläinen J., Pohjolainen S. (2010). Students' Motivations for Social Media Enhanced Studying and Learning. Knowledge Management \& E-Learning: An International Journal (KM\&EL), 2(1), $51-67$.

17 Tan, T.H., Liu, T.Y. \& Chang, C.C. (2007). Development and evaluation of an RFID-based ubiquitous learning environment for outdoor learning. Interactive Learning Environment, 15:3, 253 - 269.

18 Teo Y.H. \& Chai C.S. (2009). Scaffolding Online Collaborative Critiquing for Educational Video Production. Knowledge Management \& E-Learning: An International Journal (KM\&EL), 1(1), 51 - 66.

19 Thompson, C.W., Li, W.N. \& Xiao, Z. (2007). Workflow planning on a grid. IEEE Internet Computing, 11(1), $74-77$.

20 Vreman O.C. \& Jong T. (2006). Scaffolding learners in designing investigation assignments for a computer simulation. Journal of Computer Assisted Learning 22(1), $63-73$.

21 Yang, S.J.H. (2006) Context Aware Ubiquitous Learning Environments for Peer-toPeer Collaborative Learning. Journal of Educational Technology and Society, 9(1), $188-201$.

22 Yang, S.J.H. \& Chen I.Y.L. (2008). A social network-based system for supporting interactive collaboration in knowledge sharing over peer-to-peer network. International Journal of Human-Computer Studies, 66(1), 36-50.

23 Yang, S.J.H., Okamoto T. \& Tseng S.S. (2008). Guest Editorial: Context-aware ubiquitous learning. Educational Technology and Society, 11(2), 1-2.

24 Yang, S.J.H., Zhang J. \& Chen I.Y.L. (2008). A JESS enabled context elicitation system for providing context-aware Web services. Expert Systems with Applications, $34(4), 2254-2266$. 